\title{
Article \\ A Polygonal Model to Design and Fabricate Ion-Exchanged Diffraction Gratings
}

\author{
Carlos Montero-Orille*D, Xesús Prieto-Blanco ${ }^{(D)}$, Héctor González-Núñez $\mathbb{D}^{\mathbb{D}}$ and Jesús Liñares
}

Quantum Materials and Photonics Research Group, Optics Area, Department of Applied Physics, Faculty of Physics/Faculty of Optics and Optometry, Universidade de Santiago de Compostela, Campus Vida s/n, E-15782 Santiago de Compostela, Galicia, Spain; xesus.prieto.blanco@usc.es (X.P.-B.); hector.gonzalez.nunez@usc.es (H.G.-N.); suso.linares.beiras@usc.es (J.L.)

* Correspondence: carlos.montero@usc.es

check for updates

Citation: Montero-Orille, C.; Prieto-Blanco, X.; González-Núñez, H.; Liñares, J. A Polygonal Model to Design and Fabricate Ion-Exchanged Diffraction Gratings. Appl. Sci. 2021, 11, 1500. https://doi.org/10.3390/ app11041500

Academic Editor: Andrey

Miroshnichenko

Received: 10 December 2020

Accepted: 1 February 2021

Published: 7 February 2021

Publisher's Note: MDPI stays neutral with regard to jurisdictional clai$\mathrm{ms}$ in published maps and institutional affiliations.

Copyright: (C) 2021 by the authors. Licensee MDPI, Basel, Switzerland. This article is an open access article distributed under the terms and conditions of the Creative Commons Attribution (CC BY) license (https:// creativecommons.org/licenses/by/ $4.0 /)$.

\begin{abstract}
We propose a simple polygonal model to describe the phase profile of ion-exchanged gratings. This model enables the design of these gratings, as well as the characterization of the ion-exchange process itself. Several ion-exchanged gratings were fabricated to validate the model and to characterize the process involved in their fabrication. From this characterization, we show the practical utility of the model by designing and fabricating both a grating that removes the zero order and a three splitter. The performance of these two elements was good, although the first one stood out especially because only $0.5 \%$ of the power remained in the zero order after diffraction. This polygonal model could be useful to design more complex diffractive elements.
\end{abstract}

Keywords: ion-exchange; phase gratings; diffractive optics

\section{Introduction}

Diffractive Optical Elements (DOEs) are widely used in different fields such as holography [1], spectroscopy [2], astronomy [3], and so on. Among DOEs, diffraction gratings stand out for their versatility, being a common part of devices for wavelength division multiplexing, optical pulse compression, or laser tuning. Gratings modulate the amplitude or the phase of the light incident on them. As a result, light is diffracted into the so-called diffraction orders. In applications where the diffraction efficiency is important, phase modulation is preferred to amplitude modulation. The most investigated phase gratings are those with sinusoidal profiles. The gratings with this kind of profile have significant advantages. First, they can be mathematically analyzed by closed-form expressions. Secondly, these profiles can give rise to many interesting diffraction patterns including the cancellation of the zero order, splitting in several orders, and so on. Lastly, sinusoidal gratings can be fabricated by interference of two plane waves on photosensitive substrates. However, gratings fabricated in this way are delicate, and specific phase profiles are difficult to obtain. Here, we use the ion-exchange technique to fabricate robust and precise sinusoidal-like phase gratings by taking advantage of its simplicity, low cost, and easy control of the fabrication parameters [4]. Moreover, the flat surface of the elements fabricated by this technique permits straightforward cleaning and antireflection coating, as well as integration in stacked optical systems [5]. As is known, ion-exchange changes the refractive index of suitable substrates, typically in a region of some micrometers under its surface. This change can be made selectively in only some regions of the substrate, provided that a mask for the ions is used. In particular, by designing appropriate masks, as well as the ion-exchange process itself, different kinds of phase gratings have been fabricated [6,7]. In these works, the authors successfully designed and fabricated grating beam splitters by using a numerical approach for both the ion-exchange process and the diffraction calculations. However, the design of such elements using numerical methods is not easy when trying to make complex elements. In the present work, we propose a 
simple polygonal model for the phase profile achieved following thermal ion-exchange processes. This model enables the design and fabrication of grating elements in a simple way. After characterization of the ion-exchange process by using such a model, different grating elements can be designed and fabricated easily. As examples, we design and fabricate a diffraction grating with suppressed zero order and a three splitter. Cancellation of the zero order of diffraction is desirable in many applications of diffractive elements, such as digital holography [8], spatial light modulation [9], and grating fabrication [10], among others. On the other hand, light splitting is useful, in general, for power division tasks [11], and in particular, it has been used, combined with other phase functions, in the fabrication of vortex gratings [12].

In Section 2, we explain the characteristics of the model and obtain expressions for the transmission function of gratings with such a phase profile. Next, we describe the characterization procedure of the two-dimensional ion-exchange process by using this model. This characterization provides the characteristic parameters of the model as a function of the ion-exchange time. Finally, in Section 4, we check the potential of the model by designing and fabricating a grating with suppressed zero order and a three splitter.

\section{Polygonal Model for the Phase Profile of Ion-Exchanged Gratings}

The phase profile of a grating is conditioned by its fabrication process. In particular, in the fabrication of gratings by thermal ion-exchange through a mask, the phase profile generated is determined not only by the shape of the mask, but also by the ion-exchange process itself. As a first approach, the phase profile of a grating fabricated by this technique would be a square profile. However, when a thermal ion-exchange takes place through a mask opening, ions diffuse beneath the masked part generating a transition region (s), which extends beyond the opening $(w)$ (see Figure 1).

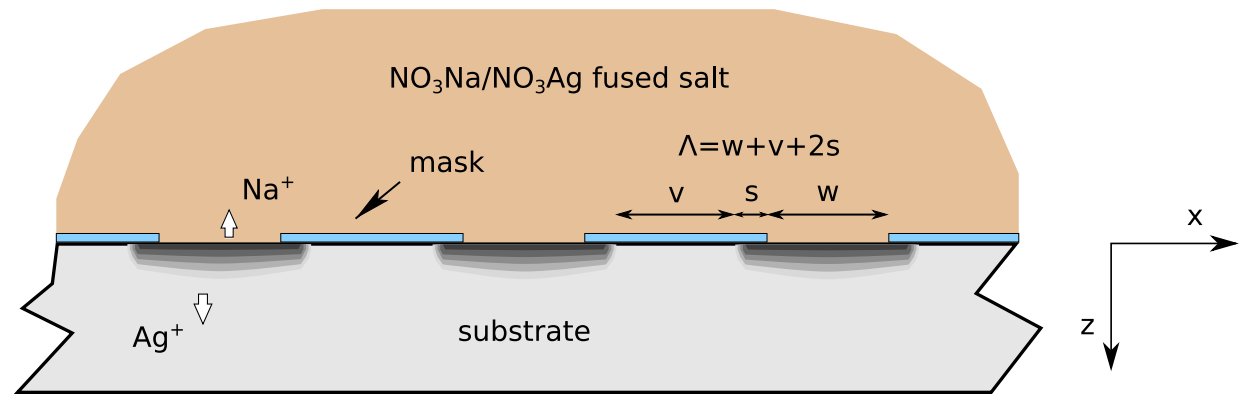

Figure 1. Scheme of the fabrication of an ion-exchanged grating. $s$ and $\Lambda$ indicate the transition region (or side diffusion) and the period of the grating, respectively.

Then, the phase profile of an ion-exchanged grating, $\Delta \varphi(x)$, would be more like a trapezoid. Moreover, the openings are usually similar in size to the transition region. This makes the ions spread deeper in the middle of the openings than close to their edges. As a result, the phase profile should be rounded in the region corresponding to the opening, just as predicted by the diffusion equation that governs the ion-exchange process [7] and confirmed experimentally [13]. In a first approximation, we can assume, for this region, a triangular phase profile and a linear profile for the transition region or side diffusion. Therefore, all the phase profile can be modeled by using straight lines giving place to the so-called polygonal model (see Figure 2).

In order to gain more insight into this shape of the phase profile, we plot, in Figure 3, typical profiles of in-depth refractive index changes of a substrate, $\Delta n(z)$, for three side positions: at the middle of the opening, at the edge of the mask, and at the middle of the transition region. As is known, this change of the refractive index is proportional to the concentration $C(x, z)$ of in-diffused ions, which can be calculated by solving a twodimensional non-linear diffusion equation [7]. From $\Delta n(x, z)$, the phase profile is calculated by [14]: 


$$
\Delta \varphi(x)=\frac{2 \pi}{\lambda} \int_{0}^{\infty} \Delta n(x, z) d z
$$

where $\lambda$ is the wavelength. From this equation, it can be easily checked that the shape of the polygonal phase profile is compatible with the curves shown in Figure 3. On the other hand, the third dimension (y-axis) has little effect on the phase profile, and hence on the grating behavior, because the diffusion length of ions is very short compared to the length of this grating dimension. In other words, the ion-diffusion is not limited in the y-direction.

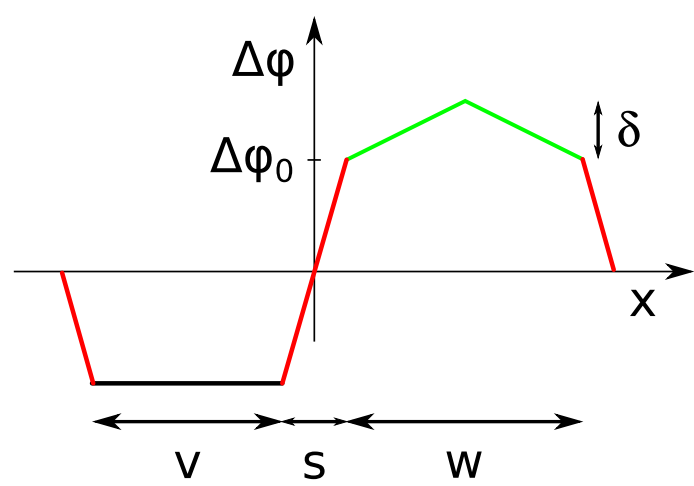

Figure 2. Modeling of the phase profile of an ion-exchanged grating by the polygonal model.

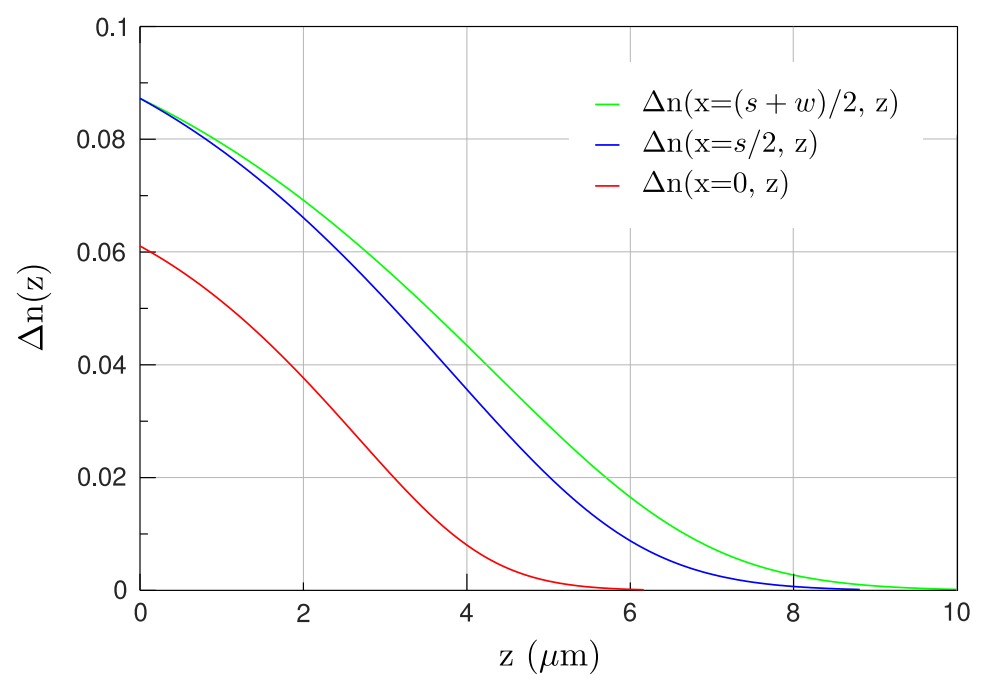

Figure 3. Typical in-depth refractive index changes after an ion-exchange process through an opening for three side positions: middle of the opening (green curve), the edge of the mask (blue line), and the middle of the transition region (red line).

According to Figure 2, the phase profile can be expressed as follows:

$$
\Delta \varphi(x)=\left\{\begin{array}{cc}
-2 \Delta \varphi_{0}(1+v / s+x / s) & -(v+s) \leq x \leq-(v+s / 2) \\
-\Delta \varphi_{0} & -(v+s / 2) \leq x \leq-s / 2 \\
2 \Delta \varphi_{0} x / s & -s / 2 \leq x \leq s / 2 \\
\Delta \varphi_{0}+\delta(-s / w+2 x / w) & s / 2 \leq x \leq(w+s) / 2 \\
\Delta \varphi_{0}+\delta(2+s / w-2 x / w) & (w+s) / 2 \leq x \leq w+s / 2 \\
2 \Delta \varphi_{0}(1+w / s-x / s) & w+s / 2 \leq x \leq w+s
\end{array}\right.
$$

where all the variables are defined in that figure. This model allows the characterization of the two-dimensional ion-exchange process, which takes place in the fabrication of the grating through the determination of the parameters of the model $\left(s, \delta\right.$, and $\left.\Delta \varphi_{0}\right)$. In Section 3, we will explain the procedure that we used. It is based on the comparison 
between the theoretical and experimental efficiencies of the gratings fabricated and will show the utility of this simple model.

For more complex periodic structures, the phase profile would be given by the following general polygonal function:

$$
\Delta \varphi(x)=\left\{a_{j}+b_{j} x, \quad x_{j-1} \leq x \leq x_{j}, \quad j=1, \ldots, M, \quad x_{0}=-\frac{\Lambda}{2}, x_{M}=\frac{\Lambda}{2}\right\},
$$

where $\Lambda$ is the period of the grating, $M$ the number of segments, and $a_{j}, b_{j}$ would be determined by the specific shape of the profile. The substitution of this expression in the equation for the transmission of the order $m$ of a grating, in the thin-grating approximation,

$$
t_{m}=\frac{1}{\Lambda} \int_{-\Lambda / 2}^{\Lambda / 2} \exp (i \Delta \varphi(x)) \exp \left(-i m \frac{2 \pi}{\Lambda} x\right) d x,
$$

leads to a general expression for the diffraction coefficients of such a grating:

$$
t_{m}=\sum_{j} \frac{\exp \left(i a_{j}\right)}{i\left(\Lambda b_{j}-2 \pi m\right)}\left\{\exp \left[i\left(b_{j}-\frac{2 \pi m}{\Lambda}\right) x_{j}\right]-\exp \left[i\left(b_{j}-\frac{2 \pi m}{\Lambda}\right) x_{j-1}\right]\right\} .
$$

We must stress that in a standard selective ion-exchange process, the parameters $a_{j}$ and $b_{j}$ will be functions of ion-exchange time $t$. As shown later, a linear dependence between these parameters and $\sqrt{t}$ is found in a first approximation, that is,

$$
a_{j}(t)=a_{j 1}+a_{j 2} \sqrt{t} \text { and } b_{j}(t)=b_{j 1}+b_{j 2} \sqrt{t},
$$

where $a_{j 1}, a_{j 2}, b_{j 1}$, and $b_{j 2}$ would be obtained after characterization of the ion-exchange process. In general, different cases are possible: $a_{j 1}=a_{j 2}=0 ; a_{j 1}=0, a_{j 2} \neq 0 ; a_{j 1} \neq 0$, $a_{j 2}=0$; and the same cases for $b_{j 1}$ and $b_{j 2}$. This allows us to analyze the temporal evolution of diffraction coefficients, $t_{m}(t)$, so that an energy transfer is observed between diffraction orders $m$, giving rise to different optical transformations. As for the profile given by Equation (2), it corresponds to the particular case $M=6$ with $b_{2}=0$, where the period $\Lambda$ equals $w+v+2 s$. In this case, by introducing Equation (2) into Equation (4) and integrating, we obtain, after a long but straightforward calculation, the following expression for the diffraction coefficient of order $m$ :

$$
\begin{aligned}
t_{m}= & \frac{s}{\Lambda} \frac{\sin \left(\Delta \varphi_{0}-\pi m s / \Lambda\right)}{\Delta \varphi_{0}-\pi m s / \Lambda}+\frac{s}{\Lambda} \exp \left(i \frac{\pi m}{\Lambda}(v-w)\right) \frac{\sin \left(\Delta \varphi_{0}+\pi m s / \Lambda-m \pi\right)}{\Delta \varphi_{0}+\pi m s / \Lambda} \\
& -\exp \left(i \frac{\pi m}{\Lambda}(v-w)\right) \frac{\sin \left(\Delta \varphi_{0}+\pi m s / \Lambda-m \pi\right)}{m \pi}+\frac{\sin \left(\Delta \varphi_{0}-\pi m s / \Lambda\right)}{m \pi} \\
& +\frac{w}{\Lambda} \exp \left(i \Delta \varphi_{0}\right) \exp \left(-i \frac{\pi m}{\Lambda}(s+w)\right)\left(\exp \left(i\left(\frac{\pi m w}{2 \Lambda}+\frac{\delta}{2}\right)\right) \frac{\sin ((\pi m w / \Lambda-\delta) / 2)}{\pi m w / \Lambda-\delta}\right) \\
& +\frac{w}{\Lambda} \exp \left(i \Delta \varphi_{0}\right) \exp \left(-i \frac{\pi m}{\Lambda}(s+w)\right)\left(\exp \left(-i\left(\frac{\pi m w}{2 \Lambda}-\frac{\delta}{2}\right)\right) \frac{\sin ((\pi m w / \Lambda+\delta) / 2)}{\pi m w / \Lambda+\delta}\right) \\
& -\frac{w}{\Lambda} \exp \left(i \Delta \varphi_{0}\right) \exp \left(-i \frac{\pi m}{\Lambda}(s+w)\right) \frac{\sin (\pi m w / \Lambda)}{\pi m w / \Lambda} .
\end{aligned}
$$

The first two lines are the transmission for $\delta=0$ and the last ones the correction to take into account the triangle defined in the interval $[s / 2, w+s / 2]$. Note that the diffraction efficiency of the order $m$ can be easily calculated as:

$$
\eta_{m}=\left|t_{m}\right|^{2}
$$




\section{Characterization of the Two Dimensional Ion-Exchange Process by the Polygonal Model}

The polygonal model presented in the previous section enables the characterization of the ion-exchange process, which takes place during the grating fabrication. From this characterization, specific grating elements can be designed and fabricated. The detailed procedure is described here, and it consists of three steps.

First, a raw characterization of the one-dimensional ion-exchange process is made through the fabrication of some planar waveguides in suitable substrates. In our case, we fabricated these waveguides by using thermal ion-exchange in soda-lime glasses and a mixture $5 \%$ mole $\mathrm{AgNO}_{3} / \mathrm{NaNO}_{3}$, at $340^{\circ} \mathrm{C}$, as the ion source. Next, the ion-exchange modification produced by this salt in this particular glass is analyzed by the inverse Wentzel-Kramers-Brillouin (IWKB) method. As is known, the IWKB method facilitates the determination of the in-depth refractive index profile resulting from the ion-exchange process. From this refractive index profile, the phase step and the penetration depth of the ions, for a given ion-exchange time, can be calculated [14]. In particular, we needed an ion-exchange time $t=13 \mathrm{~min}$ to get an in-depth penetration of about $7.5 \mu \mathrm{m}$ and a phase step around $\pi$ for a wavelength $\lambda_{0}=632.8 \mathrm{~nm}$.

Second, the geometric characteristics of the gratings are chosen. In particular, their period is usually selected from diffraction needs. We chose a period $\Lambda=30 \mu \mathrm{m}$ and an opening width $w=10 \mu \mathrm{m}$. The size of the opening was chosen to obtain a grating with the two sides of its phase profile similar in size, that is a quasi-antisymmetric phase profile. Note that most interesting phase profiles are of this kind. This opening width leads to $v \approx$ $10 \mu \mathrm{m}$ and $s \approx 5 \mu \mathrm{m}$ for a phase step $\pi$, because the side diffusion of the ions is somewhat smaller than the in-depth diffusion. Next, the grating must be patterned on the substrate. We used for it a two-step photolithographic process. In the first step, a binary grating pattern with the aforementioned dimensions was plotted on a sheet of heavy paper and then reduced $-1 / 13.5$ by using a lens Schneider XENON Sapphire 4.5/95. This led to a chromium master on a glass substrate that was then reduced by a Carl Zeiss S-planar lens working at magnification $M=-1 / 5$ and $f / \#=1.3$. The final result was an aluminum binary grating on the soda-lime glass chosen for the ion-exchange.

The third step is the characterization of the two-dimensional ion-exchange process that takes place during the grating fabrication. For that, as we will see, it is enough to use three gratings with approximate phase steps $\pi / 2, \pi$, and $3 \pi / 2$. These phase steps cover the range of phases $[0-2 \pi]$, which almost any interesting phase grating element will have. Then, from the characterization realized in the first step, we calculated the ion-exchange times ( $3.5 \mathrm{~min}, 14.5 \mathrm{~min}$, and $32 \mathrm{~min}$ ) needed to achieve, approximately, these phase steps. Next, after the fabrication of these three gratings, their diffraction efficiencies $\left(\eta_{m}^{\text {exp }}\right)$ were measured and compared to those provided by the polygonal model (Equations (7) and (8)). This is done by calculating the RMS of the differences between measured and calculated efficiencies, that is:

$$
\operatorname{RMS}\left(w, s, \delta, \Delta \varphi_{0}\right)=\sqrt{\sum_{m=-(N-1) / 2}^{(N-1) / 2}\left(\eta_{m}^{\text {exp }}-\eta_{m}^{\text {Equation }(7)}\right)^{2} / N},
$$

where $N$ is the number of the diffraction orders measured. The minimization of this RMS permitted us to fit the polygonal model to the exact phase profile of each grating. Note that in addition to the intrinsic parameters of the model, we also considered the width of the opening as a free parameter. This was done to take into account the fabrication tolerances and/or to allow the approximate model to fit better to the real profile.

We did the minimization task by using the function fmincon of the software Octave [15]. This minimization was made in two passes. In the first one, we let the four parameters vary freely, except for $w$, which we constrained to values close to the real opening width $(w=10 \pm 1.5 \mu \mathrm{m})$. After this, several findings can be highlighted. First, all three minima of the RMSs were under $0.05 \%$, which confirms the validity of the model. 
Second, $w$ varied approximately between 8.5 and $10 \mu \mathrm{m}$. Third, a good linear relationship was observed between $s$ and $\Delta \varphi_{0}$ and $\sqrt{t}$. This behavior was expected because the penetration depth in ion-exchange processes, as well as the phase steps that ion-exchange yields depend linearly on the square root of time [14]. Finally, the dependence between $\delta$ and $\sqrt{t}$ was not clearly linear. However, it is reasonable to guess a linear dependence between these two magnitudes because $\delta$ is a part of the maximum phase step $\left(2 \Delta \varphi_{0}+\delta\right)$, and this should depend linearly on $\sqrt{t}$, at least for short times. Moreover, we checked that the minima of the RMSs were not too sensitive to changes in $\delta$. With this in mind, we did a second minimization pass where the values of $\delta$ were constrained to the best linear fit obtained in the first pass $\pm 10 \%$, and $w$ was fixed to the average of the three values resulting from this first fit, that is $9.1 \mu \mathrm{m}$. Note that this value of the opening width is remarkably different from the real value $(10 \mu \mathrm{m})$ of the fabricated mask. This is a consequence of the coarse approximation of the phase profile that we obtained with the polygonal model. After the second minimization pass, the three minima of the RMSs were under $0.08 \%$, and we obtained the linear dependencies shown in Figure 4.
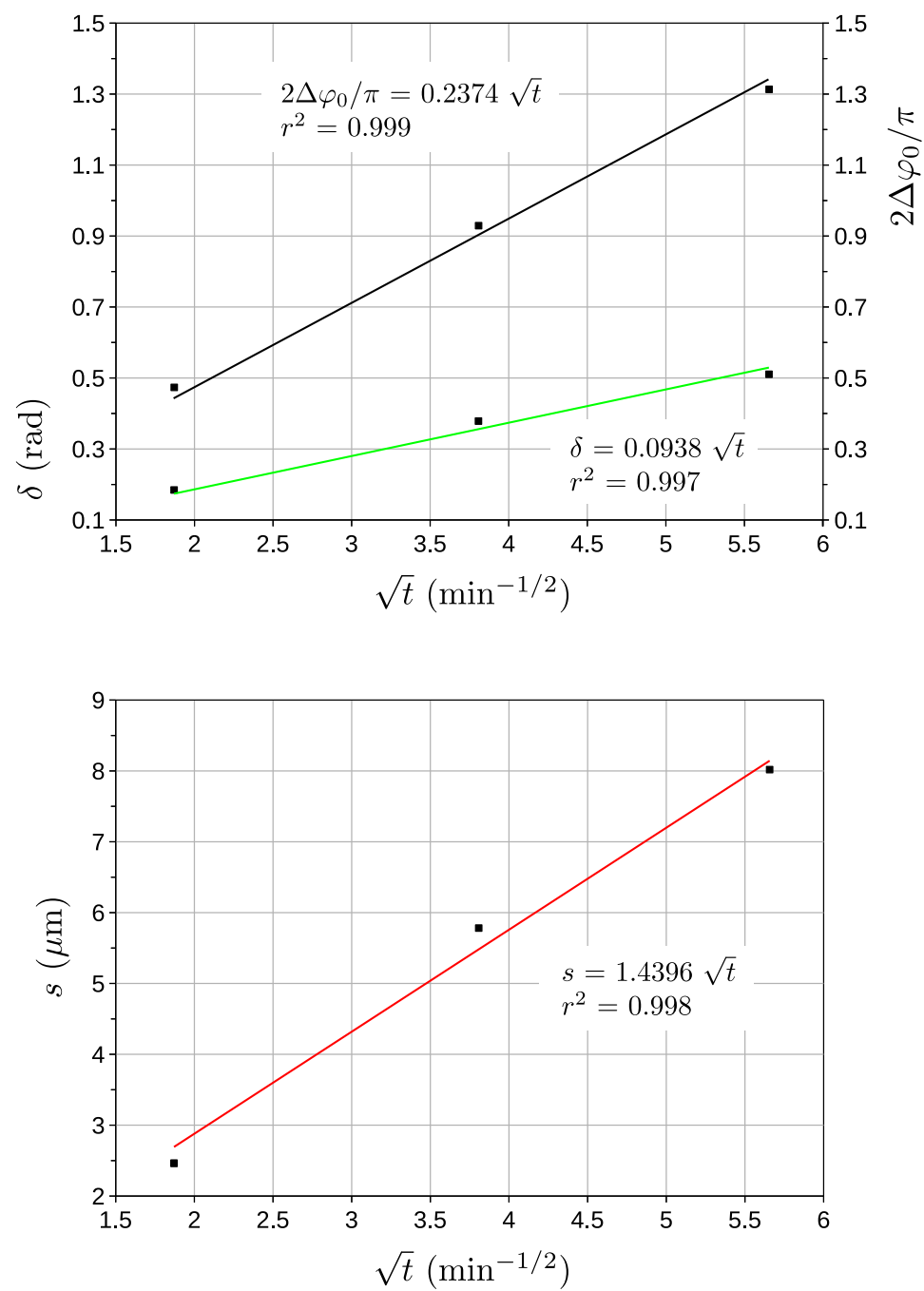

Figure 4. Data points of the polygonal model that best predicted the experimental efficiencies of the three gratings fabricated to characterize the ion-exchange process; the lines of the best fit as functions of the square root of the ion-exchange time are also plotted. The parameter $w$ was fixed to $9.1 \mu \mathrm{m}$.

This figure confirms the linear dependencies already observed in the first pass. However, a closer examination reveals a slight departure from the linearity of $\Delta \varphi_{0}$ and $\delta$. This is a consequence of the saturation of the in-depth diffusion of ions for long times. Anyway, as we will see in the next section, it did not have any apparent impact on the design and 
fabrication of, at least, the two elements that we made. On the other hand, we checked that the inclusion of more than three gratings in the above characterization did not change the quality of the fits or the predictions. Now, if we insert in Equation (7) the linear equations shown in this figure, only the ion-exchange time remains as the independent variable. Therefore, we can represent the efficiencies of the diffraction orders (Equation (8)) as functions of $\sqrt{t}$ (see Figure 5). This figure includes the efficiencies of the three gratings used in the characterization process, as well as those of the two grating elements that we designed and fabricated using such a characterization, as we will see in the next section.

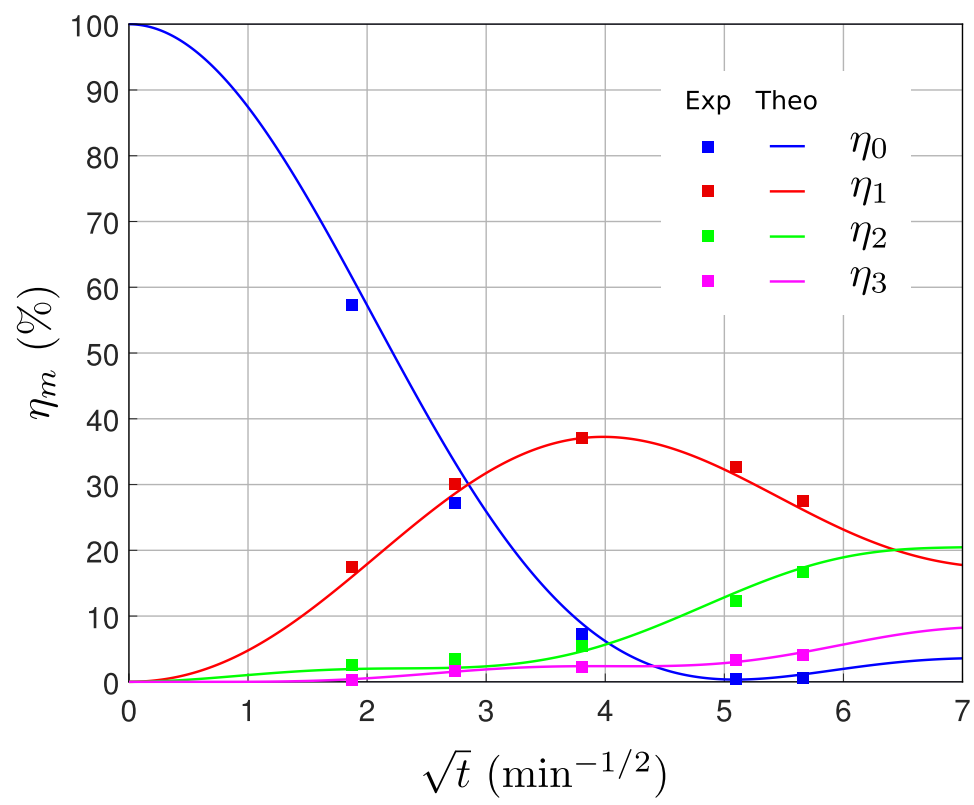

Figure 5. Plot of the efficiencies of the orders 0 (blue line), \pm 1 (red line), \pm 2 (green line), and \pm 3 (magenta line) with the square root of the ion-exchange time according to Equations (7) and (8) and Figure 4 . The experimental efficiencies of the five gratings used in this work are also plotted.

\section{Design and Fabrication of a Grating with Suppressed Zero Order and a Three Splitter}

Now, we show the practical utility of the model introduced in Section 2 by designing and fabricating two diffractive elements: a grating that suppresses its zero order and a three splitter. The procedure that we used for this was the same in both cases. First, we calculated the ion-exchange time necessary to obtain each element through Equations (7) and (8), as well as the characterization presented in Section 3 (Figures 4 and 5). Next, we fabricated such an element following the same fabrication process explained in Section 3, but using the previous calculated time. Finally, we measured the diffraction efficiencies for $\lambda_{0}=632.8 \mathrm{~nm}$ to check the quality of the element. Along these measurements, we also checked that these gratings had negligible absorption losses at this wavelength and no sensitivity to polarization of the beam. Moreover, we quantified their noise by measuring the stray light between $0-1$ orders and 1-2 orders. We obtained a signal-to-noise ratio higher than $35 \mathrm{~dB}$. The experimental setup to measure the diffraction efficiency of these grating elements is shown in Figure 6, and a close up photo of one of the fabricated gratings can be seen in Figure 7.

Of course, the design of these elements was restricted to the parameters of the gratings used to characterize the ion-exchange process, that is a period $\Lambda=30 \mu \mathrm{m}$ and an opening width $w=10 \mu \mathrm{m}$. However, other periods and openings could be used provided that a previous characterization is done. In particular, by using this polygonal model, the period and the opening of the gratings could be optimized for a specific element on the basis of, for instance, a large fabrication tolerance or another merit function. On the other hand, this model provides the minimum period that can be achieved using this technique of 
grating fabrication. From Figures 1 and 2, it is clear that the limit is found for $v=0$, that is $\Lambda_{\min }=w+2 s$. The particular value will depend on $s$, which will be determined by the particular element to be fabricated. For example, for a grating with suppressed zero order (see Section 4.1), $\Lambda_{\min } \approx 15 \mu \mathrm{m}$, and for a three splitter (see Section 4.2), $\Lambda_{\min } \approx 9 \mu \mathrm{m}$. In both cases, an opening width of $w \approx 1 \mu \mathrm{m}$ is assumed.

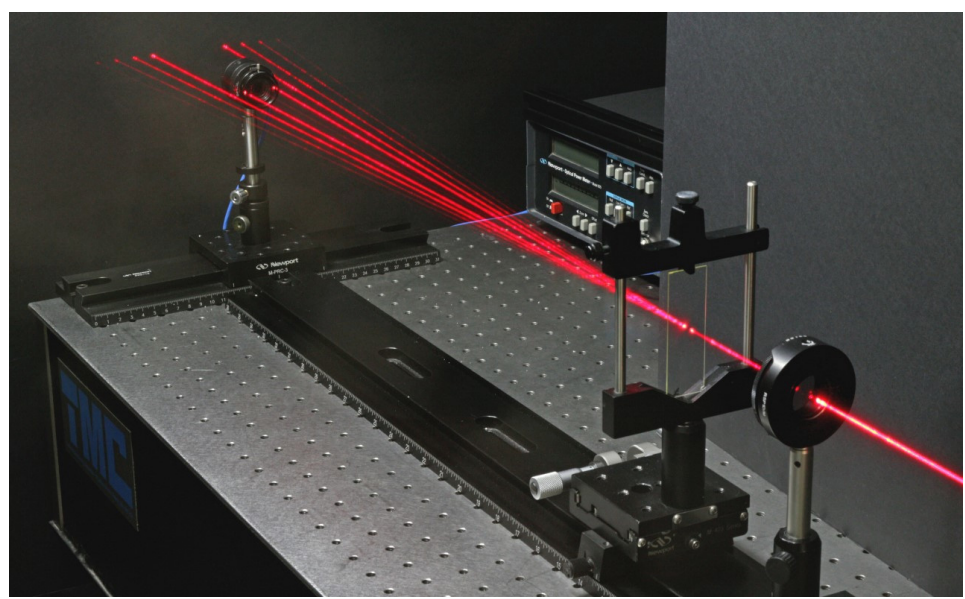

Figure 6. Long exposure photography of the experimental setup. On the right side, a polarizer, before one of the fabricated gratings, was placed to modify the intensity of the beam. On the left side, you can see the detector head, with a diaphragm on its front. The stabilized He-Ne laser used in the measurements is not shown in the picture.

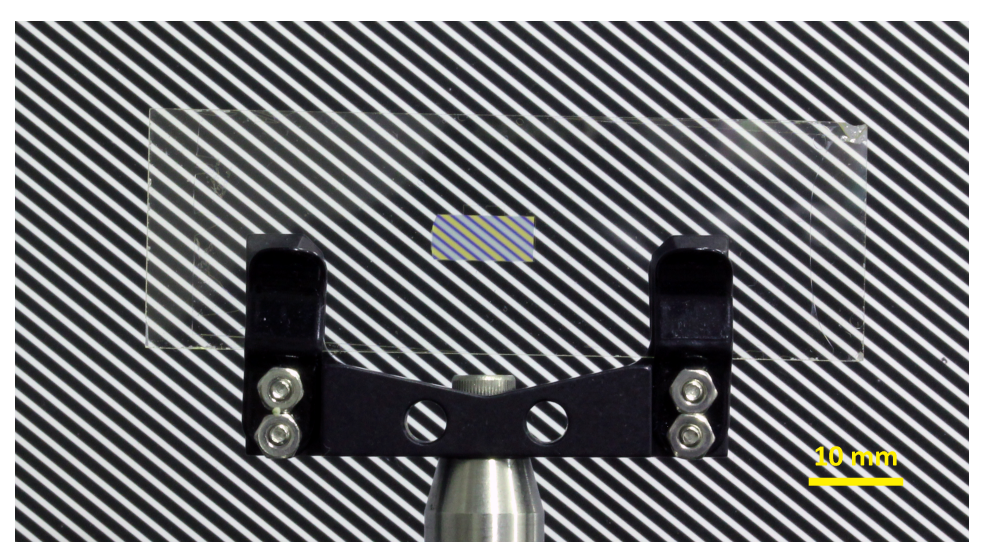

Figure 7. Close-up photography of one of the fabricated gratings (in the middle of the picture). Behind, you can see a periodic object to better observe the dimensions of the grating with respect to the glass substrate. The grating grooves are arranged horizontally.

\subsection{Design and Fabrication of a Grating with Suppressed Zero-Order}

The cancellation of the zero order implies $\eta_{0}=0$. According to the blue curve (efficiency of the zero order) of Figure 5, this condition is fulfilled for $t \approx 26 \mathrm{~min}$. Therefore, we fabricated a new grating using this ion-exchange time. After fabrication, we measured the diffraction efficiencies and obtained the results shown in Table 1. 
Table 1. Experimental and predicted diffraction efficiencies for $\lambda_{0}=632.8 \mathrm{~nm}$ of the grating designed to suppress its zero order.

\begin{tabular}{ccc}
\hline$m$ & $\left|t_{m}^{2}\right| \exp$ & $\left|t_{m}^{2}\right|_{\text {Equation(7) }}$ \\
\hline 0 & $0.5 \%$ & $0.35 \%$ \\
\pm 1 & $32.55 \%$ & $31.4 \%$ \\
\pm 2 & $12.2 \%$ & $13.6 \%$ \\
\pm 3 & $3.25 \%$ & $3.0 \%$ \\
\pm 4 & $1.3 \%$ & $1.3 \%$ \\
\pm 5 & $0.2 \%$ & $0.3 \%$ \\
\pm 6 & $0.05 \%$ & $0.05 \%$ \\
\hline
\end{tabular}

The result was very good. The efficiency of the zero order did not reach the theoretical minimum, but was very close, and the efficiency of the first orders $( \pm 1)$ was higher than expected. This closeness between the predicted and obtained values was not surprising because of the low sensitivity of the diffraction efficiency $\eta_{0}$, at its minimum, with the ion-exchange time (see Figure 5). Furthermore, the efficiency obtained at the first orders was slightly higher than the value (27\%) that a sinusoidal phase grating provides. If higher efficiencies were necessary, we could stack a second grating with half the period to obtain a non-symmetrical phase profile. A proper design of these two phase elements would result in an improved efficiency for the +1 order at the expense of the -1 order. On the other hand, the small discrepancy between the experimental and theoretical values of the efficiencies can be attributed to the polygonal model, which does not perfectly reproduce the real phase profile. Anyway, the small values $\eta_{0}$ that we obtained validate this ion-exchange technique to fabricate this kind of gratings and the polygonal model to represent its phase profile.

\subsection{Design and Fabrication of a Three Splitter}

The procedure that we used to fabricate a three splitter was the same as described in the previous paragraphs, but now the condition $\eta_{0}=\eta_{1}$ or $\eta_{0}=\eta_{-1}$ must be fulfilled. Therefore, from Figure 5, we must calculate the intersection between the blue curve (efficiency of the zero order) and the red curve (efficiency of the first order). This intersection is at $t \approx 8.1 \mathrm{~min}$; however, we finally rounded this time to $t=8 \mathrm{~min}$ because of the experimental uncertainties of the fabrication process. Indeed, for such a short time, the transient regimes are relevant, and we observed that they gave rise to uncertainties of about $3 \%$ in the ion-exchange time. After fabrication, we measured the diffraction efficiencies of the first three orders. They were $\eta_{0}=25.5 \%$ and $\eta_{ \pm 1}=31.0 \%$. It is clear from these values that our goal was not achieved. Three reasons can be given to explain such a discrepancy. First, is the inaccuracy of the polygonal model; second is the error in the opening width resulting from the photolithographic process; and third is the high sensitivity, in this region, of the efficiencies of the first two orders with the duration of the ion-exchange process, which along with the aforementioned uncertainties made the prediction very difficult. Both the second and third reasons can be minimized by using a thorough lithography and lower temperatures for the ion-exchange process, respectively. Despite the differences obtained between the efficiencies, note that the model gave a result close to the desired one, as well as clues to find a better solution. Indeed, according to Figure 5, the time was overestimated because the efficiencies obtained were on the right side of the intersection, at $t \approx 9 \mathrm{~min}$. Therefore, we considered a new ion-exchange time $t=7.5 \mathrm{~min}$, expecting to achieve a better result. The diffraction efficiencies of this last grating are shown in Table 2. The values were not yet optimal, but there was no point in doing more trails due to the reasons given before. Anyway, this result can be considered good: the difference between the efficiencies of the first three orders was small (3\%), and their sum $(87.3 \%)$ was near the theoretical maximum $(92.5 \%)$. This last fact was a consequence of the similarity between the phase profile resulting from this fabrication process and the profile necessary to have an optimal three splitter [16]. It is true that the difference between the experimental and predicted efficiencies was a little high (about $6 \%$ for the zero order). However, this 
polygonal model was not conceived of to predict exactly the shape of the phase profile and hence the efficiencies of the gratings. Our goal was to provide a simple model to design optical elements made of ion-exchanged gratings.

Table 2. Experimental and predicted diffraction efficiencies for $\lambda_{0}=632.8 \mathrm{~nm}$ of the grating designed to be a three splitter.

\begin{tabular}{ccc}
\hline$m$ & $\left|t_{m}^{2}\right|_{\text {exp }}$ & $\left|t_{m}^{2}\right|_{\text {Equation }(7)}$ \\
\hline 0 & $27.1 \%$ & $33.3 \%$ \\
\pm 1 & $30.1 \%$ & $28.65 \%$ \\
\pm 2 & $3.5 \%$ & $2.15 \%$ \\
\pm 3 & $1.6 \%$ & $1.55 \%$ \\
\pm 4 & $0.7 \%$ & $0.5 \%$ \\
\pm 5 & $0.3 \%$ & $0.25 \%$ \\
\pm 6 & $0.1 \%$ & $0.1 \%$ \\
\hline
\end{tabular}

Finally, we show in Figure 8 the image of a red pencil (left) through the grating with suppressed zero order (middle) and the three splitter (right). Of course, this result agreed with the data given in the previous tables. With regard to the grating that suppressed its zero order, only the blue component of the pencil remained at this order because the red component was completely removed. Moreover, the \pm 1 orders were the most relevant ones. As for the three splitter, the three first orders were approximately equal in brightness, and the rest of the orders almost vanished.
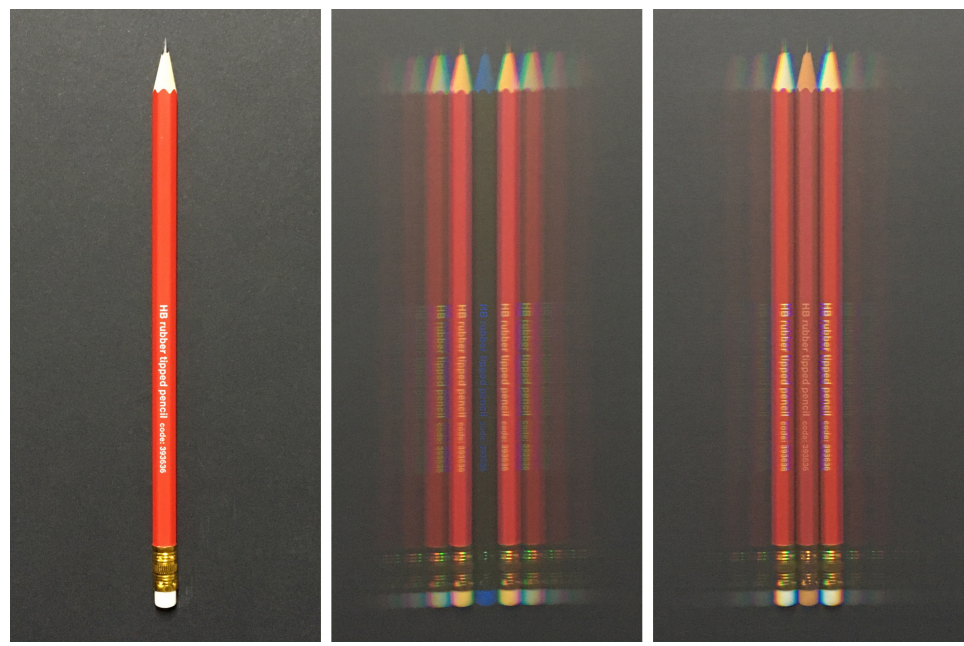

Figure 8. Images of a red pencil (left) through the grating with suppressed zero order (middle) and the three splitter (right).

\section{Conclusions}

We present a simple polygonal model to describe the phase profile of ion-exchanged gratings. Its simplicity makes it easy to calculate the transmission function of gratings with such a phase profile. From this transmission function and the experimental efficiencies of three gratings with approximate phase steps $\pi / 2, \pi$, and $3 \pi / 2$, we characterized the ion-exchange process through simple functions for the intrinsic parameters of the model. In order to show the potential of this model, we designed and fabricated a diffraction grating with suppressed zero order and a three splitter. The cancellation of the zero order was very good: only $0.5 \%$ of the power remains at this order after diffraction. As for the three splitter, its phase profile is close to the optimal one, and the difference between the efficiencies of its first orders is $3 \%$. Moreover, this value can be further improved by lowering the temperature of the ion-exchange process. Finally, this polygonal model could be useful to design more complex diffractive elements by increasing the number of segments. It could also be generalized to the two-dimensional case. 
Author Contributions: Conceptualization, X.P.-B. and J.L.; methodology, C.M.-O., X.P.-B., H.G.-N., and J.L.; software, C.M.-O. and H.G.-N.; validation, C.M.-O. and H.G.-N.; visualization, C.M.-O. and H.G.-N.; investigation, C.M.-O., X.P.-B., H.G.-N., and J.L.; writing, original draft preparation, C.M.-O.; writing, review and editing, C.M.-O., X.P.-B., H.G.-N., and J.L. All authors read and agreed to the published version of the manuscript.

Funding: This research was funded by Xunta de Galicia, Consellería de Educación, Universidades e FP, Grant GRC number ED431C2018/11, and Ministerio de Economía, Industria y Competitividad, Gobierno de España, Grant Number AYA2016-78773-C2-2-P.

Institutional Review Board Statement: Not applicable.

Informed Consent Statement: Not applicable.

Data Availability Statement: Data is contained within the article.

Conflicts of Interest: The authors declare no conflict of interest.

\section{References}

1. Hariharan, P. Optical Holography; Cambridge University Press: Cambridge, UK, 1996.

2. Gauglitz, G.; Vo-Dinh, T. Handbook of Spectroscopy; WILEY-VCH Verlag GmbH \& Co. KGaA: Weinheim, Germany, 2003.

3. Cagigal, M.P.; Valle, P.J. Wavefront sensing using diffractive elements. Opt. Lett. 2012, 37, 3813-3815. [CrossRef] [PubMed]

4. Linares, J.; Montero, C.; Moreno, V.; Nistal, M.C.; Prieto, X.; Salgueiro, J.R.; Sotelo, D. Glass processing by ion exchange to fabricate integrated optical planar components: Applications. Proc. SPIE Int. Soc. Opt. Eng. 2000, 3936, 227-238. [CrossRef]

5. Montero-Orille, C.; Moreno, V.; Prieto-Blanco, X.; Mateo, E.F.; Ip, E.; Crespo, J.; Liñares, J. Ion-exchanged glass binary phase plates for mode-division multiplexing. Appl. Opt. 2013, 52, 2332-2339. [CrossRef] [PubMed]

6. Salmio, R.P.; Saarinen, J. Graded-Index Diffractive Elements by Thermal Ion Exchange in Glass. Appl. Phys. Lett. 1995, 66, 917-919. [CrossRef]

7. Salmio, R.P.; Saarinen, J.; Turunen, J.; Tervonen, A. Graded-Index Diffractive Structures Fabricated by Thermal Ion Exchange. Appl. Opt. 1997, 36, 2048-2057. [CrossRef] [PubMed]

8. Chen, G.L.; Lin, C.Y.; Kuo, M.K.; Chang, C.C. Numerical suppression of zero order image in digital holography. Opt. Express 2007, 15, 8851-8856. [CrossRef] [PubMed]

9. Improso, W.D.G.D.; Tapang, G.A.; Saloma, C.A. Suppression of Zeroth-Order Diffraction in Phase-Only Spatial Light Modulator; Springer Series in Optical Sciences; Springer: Cham, Switzerland, 2019; Volume 222.

10. Mihailov, S.J.; Smelser, C.W.; Lu, P.; Walker, R.B.; Grobnic, D.; Ding, H.; Henderson, G.; Unruh, J. Fiber Bragg gratings made with a phase mask and 800-nm femtosecond radiation. Opt. Lett. 2003, 28, 995-997. [CrossRef] [PubMed]

11. Pöyhönen, P.; Honkanen, S.; Tervonen, A.; Tahkokorpi, M.; Albert, J. Planar 1/8 Splitter in Glass by Photoresist Masked Silver Film Ion Exchange. Electron. Lett. 1991, 27, 1319-1320. [CrossRef]

12. Marco, D.; Sánchez-López, M.M.; Cofré, A.; Vargas, A.; Moreno, I. Optimal triplicator design applied to a geometric phase vortex grating. Opt. Express 2019, 27, 14472-14486. [CrossRef]

13. Singer, W.; Dobler, B.; Schreiber, H.; Brenner, K.H.; Messerschmidt, B. Refractive-index measurement of gradient-index microlenses by diffraction tomography. Appl. Opt. 1996, 35, 2167-2171. [CrossRef]

14. Prieto-Blanco, X.; Montero-Orille, C.; Moreno, V.; Mateo, E.F.; Liñares, J. Chromatic characterization of ion-exchanged glass binary phase plates for mode-division multiplexing. Appl. Opt. 2015, 54, 3308-3314. [CrossRef] [PubMed]

15. Eaton, J.W.; Bateman, D.; Hauberg, S.; Wehbring, R. GNU Octave, Version 4.4.0 Manual: A High-Level Interactive Language for Numerical Computations. Network Theory Limited. 2018. Available online: https:/ / octave.org/doc/v4.4.1/ (accessed on 2 June 2020).

16. Gori, F.; Santarsiero, M.; Vicalvi, S.; Borghi, R.; Cincotti, G.; di Fabrizio, E.; Gentili, M. Analytical derivation of the optimum triplicator. Opt. Commun. 1998, 157, 13-16. [CrossRef] 